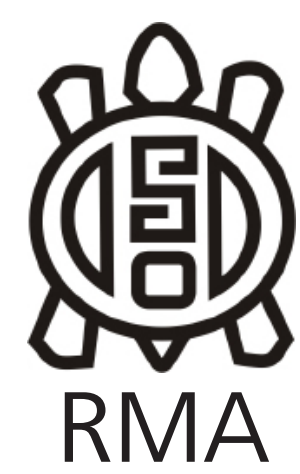

Dossier

\title{
Materias primas alfareras en dos componentes del primer milenio de la Era en El Sunchal (Anfama, Tucumán)
}

\author{
Pottery raw materials in two diachronic components of the first \\ millennium A.D. in El Sunchal (Anfama, Tucumán)
}

Francisco Franco*

*Instituto de Estudios Históricos - Consejo Nacional de Investigaciones Científicas y Técnicas (CONICET), Córdoba, Argentina. E-mail: franfranco@unc.edu.ar

\begin{abstract}
Resumen
En este aporte se presentan resultados de estudios experimentales, petrográficos y de difracción de rayos $X$ $(D R X)$ tendientes a determinar el origen de las materias primas utilizadas por los alfareros de El Sunchal (Anfama, Tucumán) durante el primer milenio de la Era. Para ello, se ha identificado la ubicación de probables fuentes de aprovisionamiento en el valle, evaluando la factibilidad de utilización y calidad de distintas vetas mediante la realización de muestras experimentales. Las briquetas resultantes de la experimentación se compararon con las pastas arqueológicas de los grupos tecnológicos principales, a partir de la observación petrográfica de veintidós cortes delgados, y de DRX de siete fragmentos. Los resultados obtenidos permitirían plantear la proveniencia local de las materias primas utilizadas.
\end{abstract}

Palabras clave: Arqueología experimental; Práctica alfarera; DRX; Petrografía cerámica.

\begin{abstract}
This contribution presents results of experimental, petrographic and X-ray diffraction (XRD) studies aimed at determining social practices linked to obtaining raw materials used by potters of El Sunchal (Anfama, Tucumán) during the first millennium A.D. For these purposes, the location of probable sources of supply in the valley has been identified, evaluating the feasibility of use and quality from the realization of experimental samples. The briquettes resulting from the experimentation were compared with the archaeological pastes of the main technological groups, based on the petrographic observation of twenty-two thin cuts, and seven fragment XRD. The results obtained would allow us to state the local origin of the raw materials used.
\end{abstract}

Keywords: Experimental archaeology; Pottery; XRD; Ceramic petrography.

En este trabajo se analizan las materias primas alfareras utilizadas en el sitio arqueológico El Sunchal (Anfama, Tucumán) durante el primer milenio de la Era (figura 1). Para ello, a nivel teórico se parte de los desarrollos de la Arqueología de la Práctica, los cuales permiten abordar al saber técnico y a su práctica como principios subjetivos pero no individuales, generadores y organizadores de las prácticas y representaciones cotidianas (Pauketat 2001; Bourdieu 2007).

El abordaje desde la práctica permite, en sentido bidireccional, pensar en un habitus estructurante, que se expresa como una tradición técnica en el sentido otorgado por Cremonte (1996), y que da forma a prácticas tecnológicas reiteradas en el tiempo. Pero también, donde la tecnología da contenido a determinadas formas estructurales, ya que la acción nunca resulta idéntica a su predecesora, generando (re) actualizaciones permanentes, que en ocasiones pueden ser observadas en el registro arqueológico (Dobres y Hoffman 1994; Bourdieu 2007; García Roselló 2009; Feely 2010; Di Prado 2018).

En trabajos clásicos del área sudcalchaquí, se ha considerado a la práctica alfarera como un sistema estable a lo largo del primer milenio (Berberián y Arguello 1988) o una tradición (Cremonte 1996 y 2003), enfatizando mayormente la dimensión de perdurabilidad de las prácticas, por sobre sus aspectos variables. Esto se manifiesta en la presencia de similitudes morfoestilísticas en la alfarería de un amplio sector de valles y piedemonte (Scattolin 2006a; Scattolin et al. 2007; Maldonado et al. 2011) las cuales serían extensibles a las actuales provincias de Tucumán (oeste y noroeste), Catamarca (norte y noreste), y Salta (sur) ${ }^{1}$. En adición, numerosos

\footnotetext{
${ }^{1}$ Incluyo dentro de esta delimitación a los valles occidentales del sector sur de las cumbres Calchaquíes (Tafí, Yocavil), así como a los faldeos y piedemontes orientales (Anfama, Sierra de San Javier), aunque la
} 


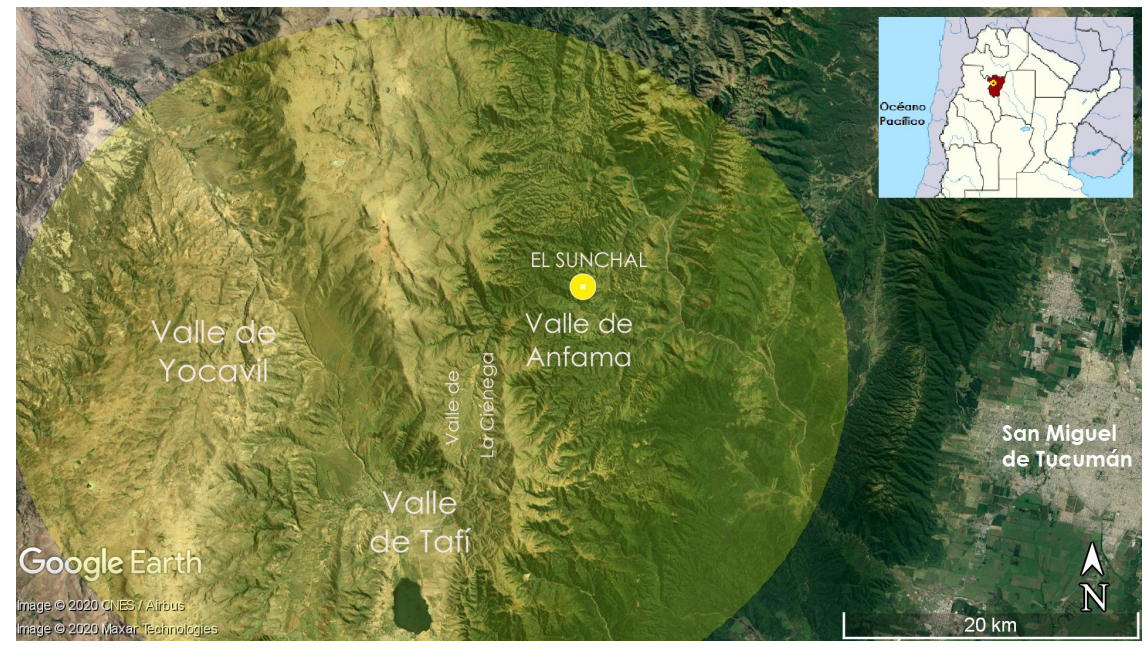

Figura 1. Ubicación de El Sunchal en la provincia de Tucumán (Argentina). En amarillo, sector denominado como "área sudcalchaquí".

Figure 1. Location of El Sunchal (Tucumán, Argentina). In yellow, sector referred as "sudcalchaqui area".

autores han planteado que el origen de las materias primas habitualmente utilizadas para la producción alfarera sería eminentemente local durante el primer milenio d.C. (Srur 1998 y 1999; Cremonte 1996 y 2003; Scattolin 2007; Pereyra Domingorena 2012 y 2015; Lazzari et. al. 2016 y 2017; Giusta 2017; entre otros).

El objetivo de este trabajo es dialogar con los trabajos precedentes, a partir de nuevos datos obtenidos para El Sunchal en relación a las materias primas utilizadas en la cerámica. El sitio presenta una serie de eventos constructivos y ocupacionales diacrónicos, evidenciados por variables arquitectónicas y fechados radiocarbónicos (figura 2). La principal complejidad que presenta la locación es que la mayor parte de las estructuras se encuentran soterradas, con lo cual los avances tendientes a determinar con mayor precisión la cantidad y tipo de ocupaciones se encuentran supeditadas a la realización de excavaciones en área, análisis de matrices estratigráficas y dataciones absolutas; por este motivo las primeras caracterizaciones para El Sunchal se han centrado en el análisis de la relación entre temporalidad y materialidad en relación al mismo sitio y a otras ocupaciones prehispánicas del valle de Anfama (Salazar et al. 2019).

Una línea más específica de estudios se ha centrado en la indagación de prácticas cerámicas en clave temporal comparativa (Franco 2019a y 2019b), en tanto la presencia de múltiples procesos de ocupación se configura como un campo idóneo para este tipo de análisis. Al respecto, los conjuntos artefactuales aqui considerados fueron obtenidos de dos ocupaciones residenciales temporalmente diacrónicas, y espacialmente superpuestas. La primera corresponde a un recinto circular delimitado por piedras lajas clavadas en el loess, datado por AMS en ca. 1900 años AP -cuyo piso de ocupación se denominó como A-; y la segunda a un recinto sub-rectangular de características informales, datado por AMS en ca. 1100 años AP -cuyo piso de

extensión de un universo estilístico relativamente común en la alfarería podría ser más amplio (Scattolin 2006 y Scattolin et al. 2007). los conjuntos de ambos pisos ( $\mathrm{N}$ fragmentos piso $\mathrm{A}=688$ y $\mathrm{N}$ fragmentos piso $\mathrm{B}=2286$ ) dio cuenta mayormente $(-80 \%)$ de fragmentos de paredes intermedias a gruesas (de $3 \mathrm{~mm}$ a $13 \mathrm{~mm}$ de grosor) con cocciones oxidantes u oxidantes incompletas y escasas decoraciones; los mismos fueron parte de vasijas no restringidas de contornos simples, restringidas de contornos simples, y restringidas de contornos compuestos e inflexionados. También se han identificado en menor proporción (-20\%) fragmentos finos ${ }^{2}$ (paredes menores a $3 \mathrm{~mm}$ ) y/o con cocciones reductoras $(-5 \%)$.

Un grupo minoritario de fragmentos (-10\%) presenta decoraciones incisas con motivos geométricos, aplicaciones al pastillaje, marleados (solo en el piso B), y engobes de color rojo (para mayores precisiones de los conjuntos a nivel morfoestilístico véase Franco 2019a y 2019b). Los conjuntos serían asignables a funciones de carácter cotidiano/doméstico, y presentan similitudes con los de sectores aledaños y coetáneos como La Ciénega, Tafí del Valle, Yocavil, El Cajón y San Javier (González y Núñez 1960; Heredia 1974; Berberián y Arguello 1988; Cremonte 1996 y 2003; Palamarczuk et al. 2007; Salazar et al. 2008; Bugliani 2010; Maldonado et al. 2011; Giusta 2017; entre otros).

La presencia de ambos componentes ha permitido comparar las características de arcillas y arenas en dos momentos distintos. Para ello se presentan resultados de estudios experimentales, petrográficos, y de difracción de rayos $X(D R X)$ tendientes a determinar pervivencias y variaciones en los sectores de obtención. En primer lugar, se identificaron probables fuentes de aprovisionamiento en el valle, evaluando, a partir de la realización de muestras experimentales, la factibilidad de utilización, la

\footnotetext{
${ }^{2}$ El tamaño de las inclusiones es el criterio que, por lo general, se utiliza para distinguir entre tipos finos y gruesos. Sin embargo, esa diferenciación resultó de difícil aplicación por la medida relativamente homogénea (de mediana a gruesa) de las mismas en el piso A. Por ese motivo, se utilizó como criterio de evaluación el grosor de las paredes y de las características texturales del fragmento, integrando al tamaño de las inclusiones dentro de este último punto.
} 

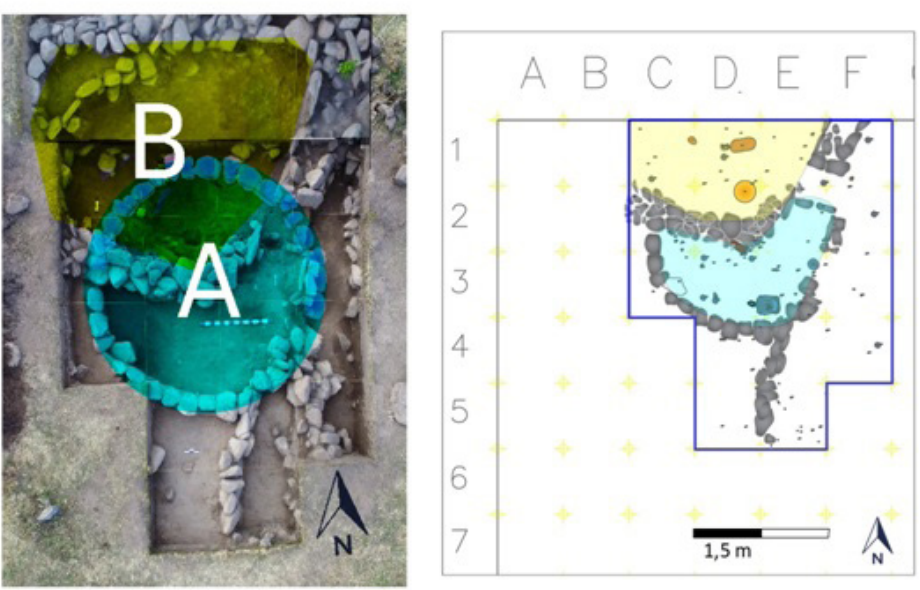

Figura 2. A la izquierda, detalle de las estructuras superpuestas. En celeste reconstrucción del piso A, en amarillo reconstrucción del piso B. A la derecha, sectores analizados de los recintos.

Figure 2. On the left, detail of the overlapping structures. In light blue, reconstruction of the $A$ floor, in yellow reconstruction of the $B$ floor. On the right, analyzed sectors of the enclosures.

Olga Tarditti en una escala cualitativa -de peor a mejor: No Modelable, Casi Nula, Muy Difícil, Difícil, Regular, Buena, Muy Buena(tabla 1). Con el material se formaron calidad de las arcillas (entendida como las características de maleabilidad y plasticidad del material), y la granulometría de las arenas. Para complementar dichos estudios, se han comparado las pastas arqueológicas de los grupos tecnológicos mayoritarios, a partir de la observación petrográfica de veintidós cortes delgados (incluyendo tres muestras experimentales), y de DRX de siete fragmentos (incluyendo tres experimentales), con el ambiente geológico de la región.

\section{Análisis experimentales}

Para la realización de esta etapa se tomaron muestras de sedimentos arcillosos de distintos sectores del valle de Anfama, los cuales fueron: Bajo Los Campitos, Bajo La Vitalia, Camino Ancho, Casa Rudi, El Duraznillo, El Sunchal, La Hoyada; del piso arqueológico B; y también de arenas del arroyo El Sunchal y del río Anfama (figura 3).

La experimentación se realizó tomando como parámetro experiencias similares realizadas por otros investigadores (p.e. García 1993; Cremonte 1996; Piñeiro 1996; entre otros). En el caso de las arcillas, las muestras fueron molidas con morteros manuales, se hidrataron y dejaron en reposo, para proceder luego a su modelado. La plasticidad y maleabilidad fue evaluada por la ceramista pequeñas barras o briquetas de $10 \mathrm{~cm}$ de largo, que contaron con distintas proporciones de agregado de las arenas recolectadas. Luego de 72 horas de secado fueron cocidas en horno eléctrico a $750{ }^{\circ} \mathrm{C}$ (1 hora de quema a ese pico de temperatura).

La arcilla obtenida en la veta de El Sunchal mostró un muy buen comportamiento, aún con adición de elevados porcentajes de arena. En tanto, las muestras obtenidas en Bajo Los Campitos, Camino Ancho (en dos sectores), Casa Rudi (en un sector) y una obtenida en El Duraznillo, mostraron buenas condiciones plásticas sin agregado de inclusiones. Por el contrario, las muestras de Bajo La Vitalia, Casa Rudi (en un segundo sector), La Hoyada, El Duraznillo (en un segundo sector), y la recuperada en el piso arqueológico B mostraron muy baja capacidad de modelado. En este último caso, corresponde marcar que la presencia de sedimentos arcillosos es habitual en los pisos de ocupación de El Sunchal, estos han sido interpretados tentativamente como parte de la cobertura de los techos de las viviendas, la escasa maleabilidad del material tal vez implique que no fuera especialmente seleccionado.

Los resultados de la experiencia marcaron una alta variabilidad inter vetas en relación a la calidad de los materiales. Estas diferencias imperceptibles a simple

Figura 3. A) Detalle de las fuentes de arcilla identificadas, en amarillo ubicación de El Sunchal, el área naranja refleja la extensión aproximada de la formación geológica Río Sali (Segemar 2014). B) Detalle de extracción de muestra en la fuente El Duraznillo.

Figure 3. A) Detail of the clay sources identified, in yellow location of El Sunchal, the orange area reflects the approximate extent of the Sali River geological formation (Segemar 2014). $B$ ) Detail of sample extraction at the El Duraznillo.

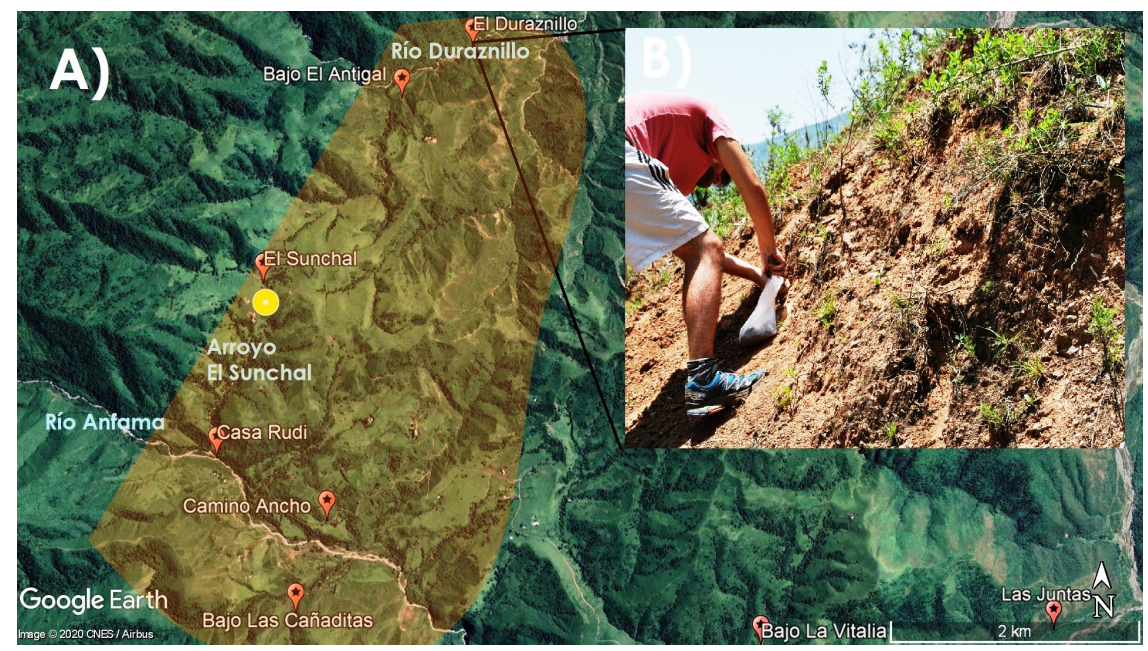




\begin{tabular}{|c|c|c|c|c|c|}
\hline Muestra & Arcilla & Arena & $\%$ & Plasticidad & Maleabilidad \\
\hline 1 & El Sunchal & El Sunchal & 10 & Muy buena & Muy buena \\
\hline 2 & El Sunchal & El Sunchal & 20 & Muy buena & Regular \\
\hline 3 & El Sunchal & El Sunchal & 30 & Muy buena & Dificil \\
\hline 4 & El Sunchal & El Sunchal & 50 & Muy buena & Muy dificil \\
\hline 5 & El Sunchal & Río Anfama & 10 & Muy buena & Muy buena \\
\hline 6 & El Sunchal & Río Anfama & 20 & Muy buena & Muy buena \\
\hline 7 & El Sunchal & Río Anfama & 30 & Muy buena & Muy buena \\
\hline 8 & El Sunchal & Río Anfama & 50 & Muy buena & Regular \\
\hline 9 & El Sunchal & Rio Anfama & 60 & Muy buena & Dificil \\
\hline 10 & El Sunchal & - & - & Muy buena & Muy buena \\
\hline 11 & Arqueológico UE72 & - & - & Casi nula & No modelable \\
\hline 12 & Camino Ancho 1 & - & - & Buena & Buena \\
\hline 13 & Camino Ancho 2 & - & - & Casi nula & No modelable \\
\hline 14 & Camino Ancho 3 & - & - & Buena & Buena \\
\hline 15 & B. Los Campitos & - & - & Buena & Buena \\
\hline 16 & B. El Antigal & - & - & Baja & Regular \\
\hline 17 & Casa Rudi 1 & - & - & Casi nula & No modelable \\
\hline 18 & Casa Rudi 2 & - & - & Casi nula & No modelable \\
\hline 19 & El Duraznillo 1 & - & - & Buena & Buena \\
\hline 20 & El Duraznillo 2 & - & - & Muy baja & No modelable \\
\hline 21 & B. La Vitalia & - & - & Casi nula & No modelable \\
\hline 22 & La Hoyada & - & - & Muy baja & No modelable \\
\hline
\end{tabular}

vista, posiblemente influyeron en las preferencias de los alfareros y alfareras por utilizar los materiales de determinadas ubicaciones y no de otras. También se detectaron diferencias en las características plásticas a nivel intra veta, lo cual se observó en los casos de El Duraznillo y de Casa Rudi. Allí, muestras de calidad heterogénea se encuentran a una distancia inferior a los 100 metros entre sí. Para un alfarero experto las diferencias de los materiales pudieron ser evidentes, mientras que para un aprendiz tal vez no lo fueran, como han planteado y evidenciado numerosos estudios centrados en el aprendizaje de la práctica alfarera (p.e. Schiffer y Skibo 1997; Crown 2001; Calvo Trias et al. 2015; Vidal 2019).

En el caso de las arenas, las obtenidas en el Arroyo El Sunchal mostraron una granulometría poco adecuada para el uso, se trata de arena muy gruesa con excesiva presencia de cantos (según escala de Wentworth 1922), la cual genera agrietamientos y fracturas en bajas proporciones de agregado. Por el contrario, la obtenida en el río Anfama, de granulometría más fina -de arena fina a arena muy gruesa- permitió el modelado, aun siendo agregada en elevadas proporciones $(+50 \%$ de la pasta). De lo observado, se desprendería que el límite de tolerancia de agrietamientos de las pastas realizadas con un buen material plástico oscilaría entre los tamaños de cantos pequeños $(2 \mathrm{~mm})$ a cantos $(4 \mathrm{~mm})$, siendo factible trabajar con granulometrías menores aún en proporciones elevadas en relación a la matriz arcillosa.

Sintéticamente, tanto para el caso de las arcillas como de las arenas, se comprobó la presencia local de materias primas adecuadas para la práctica alfarera. A partir de los resultados de esta primera etapa, se procedió a análisis más exhaustivos de la materialidad arqueológica desde perspectivas comparativas.
Tabla 1. Evaluación de plasticidad y maleabilidad de las distintas muestras obtenidas, y en relación al agregado de distintas proporciones de arena.

Table 1. Evaluation of plasticity and malleability of the different samples obtained, and mixed with different proportions of sand.

\section{Análisis Petrográficos}

La caracterización petrográfica ha sido una herramienta de análisis cerámico profusamente desarrollada en las últimas décadas para el abordaje de tradiciones prácticas y determinación de savoir faires alfareros (Cremonte 1996; Cremonte y Bugliani 2009; Pereyra Domingorena 2012; Giusta 2017; Pérez Pieroni 2017; entre otros).

En el presente trabajo, para la realización los análisis petrográficos, y con anterioridad a la observación de cortes delgados, se realizó una división en clases de pasta a partir de observación macroscópica y con lupa de bajos aumentos del total del conjunto cerámico recuperados ( $N$ piso $A=688$; $N$ piso $B=2286$ ). De ella se seleccionaron diecinueve muestras abarcando la mayor parte de los quince grupos tecnológicos identificados (tabla 2), diez de las cuales corresponden al piso A, nueve al piso $B$ y tres a briquetas experimentales (sin agregado intencional de antiplásticos). En relación a este último grupo, se seleccionaron las realizadas con materiales procedentes de las fuentes de El Sunchal (corte 8), Casa Rudi (corte 11) y El Duraznillo (corte 12), debido a que habían presentado las mejores condiciones de maleabilidad y plasticidad en la fase de experimentación.

La observación se realizó con 25 y 100 aumentos mediante un microscopio Leica DM con polarizador, se determinó: tipo, distribución y proporción de inclusiones, proporción de matriz arcillosa y cavidades, tamaño promedio de inclusiones y esfericidad de las mismas (Franco 2019a y 2019b). Los porcentajes fueron obtenidos por estimación ocular siguiendo las tablas porcentuales propuestas por Compton (1962).

Los resultados muestran, para el piso A, la presencia en todos los fragmentos de litoclastos ígneos en porcentajes que van desde un mínimo aproximado de $2 \%$, hasta un máximo de $17 \%$ del total de la pasta. En adición, se observa en tres casos la presencia de litoclastos sedimentarios (5 a 10\%). En tanto, para la ocupación B se observa una variación en el tipo de litoclastos presentes, en seis de estos cortes los litoclastos son de origen sedimentario (5 a 15\%) -dos de los cuales también contienen litoclastos metamórficos (20 a $25 \%)-$, y en solo un caso se presentan litoclastos ígneos (5\%). 
Tabla 2. Clasificación de grupos tecnológicos según características texturales.

Table 2. Classification of ceramic technological groups according to textural characteristics.

En lo que respecta a los clastos minerales, en todos los casos se constata la presencia de cuarzo, y en 21 de los 22 cortes realizados la presencia de biotita y moscovita. Las principales diferencias observadas aquí responden a la casi total ausencia de feldespato potásico en los fragmentos del piso

A, y a su mayor presencia en el piso B (5 casos de 9); y en segundo lugar a la mayor variedad de cristaloclastos observados en la ocupación temprana, presentándose granate, hornblenda y minerales opacos (si bien en cantidades mínimas), los cuales no se presentan en los fragmentos del grupo $B$, reforzando la posibilidad del cambio de arenas utilizadas (figuras 4 y 5).

Si se considera la geología local de Anfama, se observa que no es una área de presencia de rocas ígneas (Carta Geológica Segemar 2014) pese a su presencia en todas las pastas arqueológicas del piso A, de lo cual se desprende como hipótesis más probable la utilización de arenas del río Anfama, que en su cauce desde el valle de
La Ciénega arrastra material granítico desde aquel sector, y lo cual sería consecuente con la obtención local de los antiplásticos utilizados en las cerámicas del piso A.

Adicionalmente, fueron observadas muestras de arena del río Anfama en lupas de bajos aumentos, constatándose la presencia de litoclastos graníticos, cuarzo, biotita, moscovita, plagioclasa y feldespato elementos presentes mayormente en los fragmentos del piso A. En el caso de los antiplásticos identificados en los ceramios del piso $B$, se constata la presencia de formaciones geológicas consistentes con todos ellos, aunque en un área de captación potencialmente más amplia.

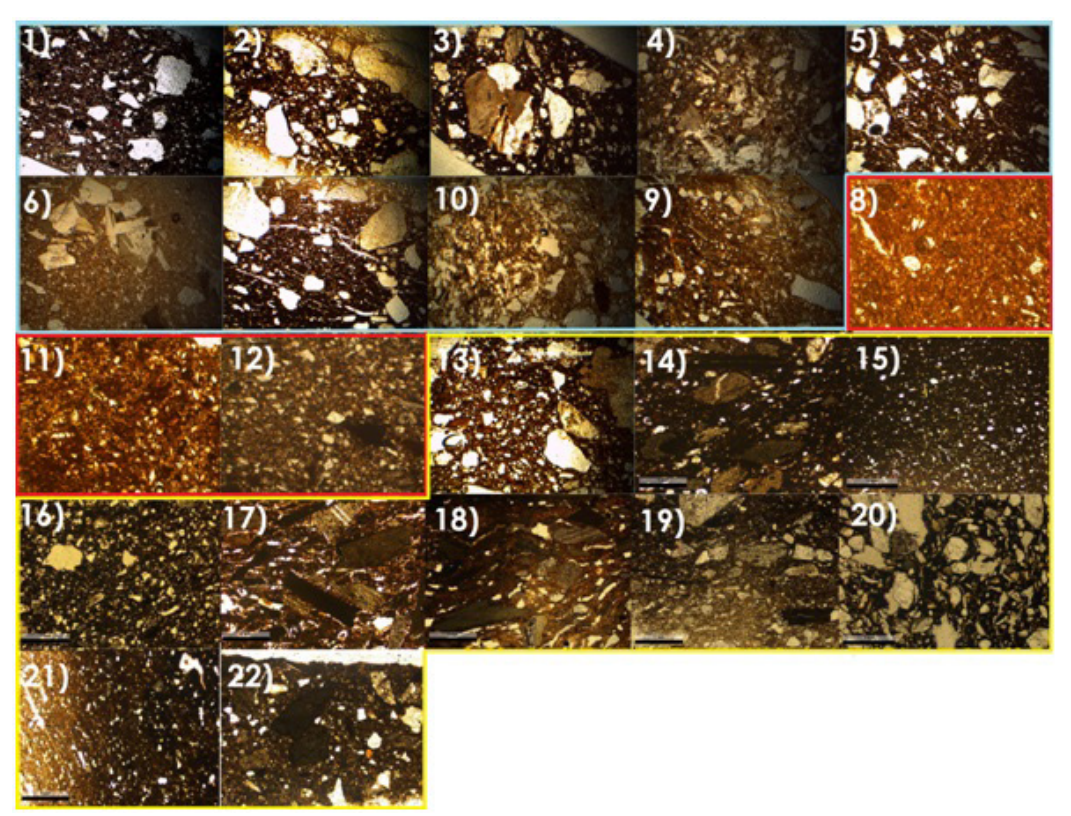

La comparación entre los tiestos de A y $\mathrm{B}$ indicaría un cambio en las arenas utilizadas, y con ello de los sectores en donde estas eran obtenidas. Además, las inclusiones de la cerámica del piso A se muestran más homogéneas a nivel

Figura 4. Detalle en nicoles paralelos de los cortes delgados realizados. Los enmarcados en celeste corresponden al piso $A$, en naranja a briquetas experimentales, y en amarillo al piso B.

Figure 4. Detail in parallel channels of the thin sections. Those framed in light blue correspond to floor $A$, in orange to experimental briquettes, and in yellow to floor $B$. 


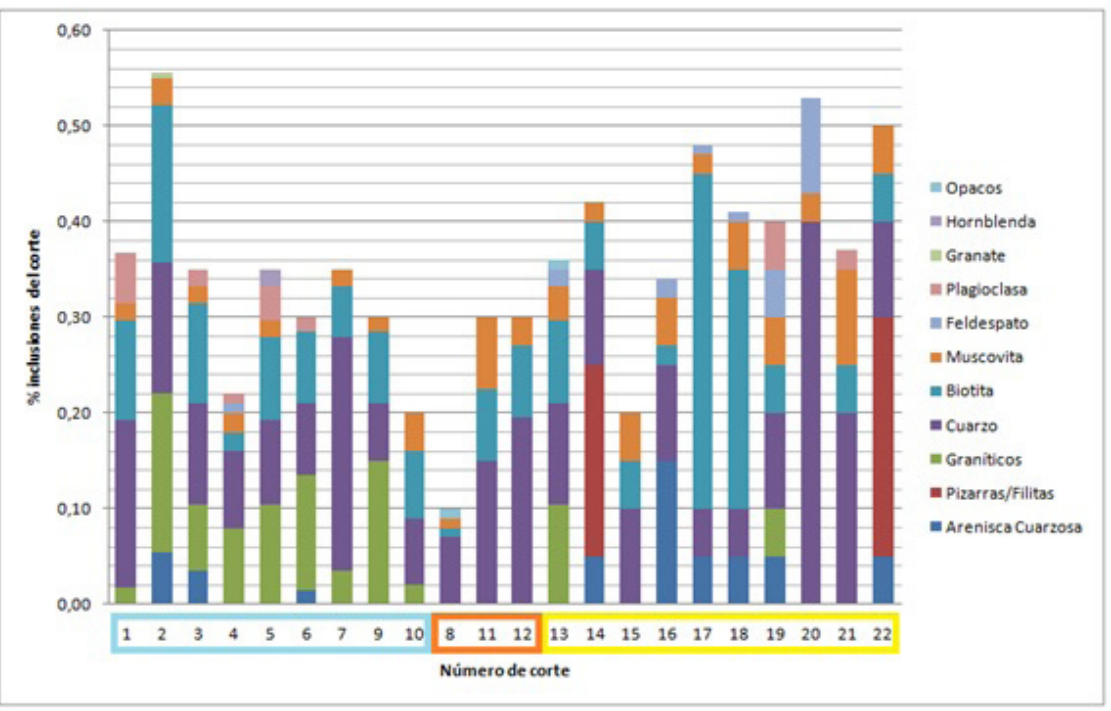

Figura 5. Proporción mineralógica de inclusiones observadas. En el eje $X$ las muestras enmarcadas en celeste corresponden al piso $\mathrm{A}$, en naranja a experimentales y en amarillo al piso B. En el eje $Y$ se detalla la proporción de cada mineral en relación al total de la matriz del corte.

Figure 5. Mineralogical proportion of inclusions observed. On the $X$ axis the samples framed in light blue correspond to the A floor, in orange to experimental and in yellow to the $B$ floor. On the $Y$ axis the proportion of each mineral is detailed in relation to the total of the thin section matrix.

de composición mineral que las observadas del piso $B$, lo que implicaría la presencia de un área relativamente estable de obtención de arenas para el primer momento, y una mayor variabilidad para la segunda fase de ocupación.

En los fragmentos experimentales (sin agregado intencional de antiplásticos), se observa casi totalmente la presencia de cuarzo, biotita, moscovita, y la ausencia absoluta de litoclastos, lo cual también se observó en fragmentos finos del piso B. Esto sería correspondiente con la manufactura de fragmentos sin agregado intencional de inclusiones durante el segundo momento ocupacional.

En las muestras experimentales se ha observado la presencia de cristales minerales de tamaño fino $(X<0,1$ $\mathrm{mm}$ ) en proporciones variables del 5\% al 30\% de la pasta. Resultaría imposible por el momento determinar por debajo de 0,1 $\mathrm{mm}$ qué porcentaje de esas inclusiones serían naturales y cuáles resultado de la acción humana, pero sí se podría plantear como hipótesis que las mayores a ese tamaño serían resultado de agregados intencionales de arena.

En adición, se constató una variación en el tamaño de las inclusiones presentes en las pastas de ambos momentos. En el caso de los fragmentos del piso A se observa la presencia de inclusiones de tamaño mínimo superior $(0,3 \mathrm{~mm})$ a las que se observan en el piso $B$ $(-0,1 \mathrm{~mm})$. La utilización de arenas más finas y la mayor variabilidad detectada en la composición de las mismas, podría indicar una mayor selección del material durante la segunda fase de ocupación.

\section{Análisis de Difracción de Rayos X (DRX)}

La realización de análisis cristalográficos también ha sido comúnmente utilizada para evaluar sectores de aprovisionamiento de materias primas (García 1993; Cremonte 1996; Piñeiro 1996; Pereyra Domingorena
2013; Schuster 2014). En este aporte se utilizaron dos metodologías complementarias para la indagación de las pastas: análisis de roca total y de fracciones $<2 \mu \mathrm{m}$. Para ambas se utilizó un difractómetro PANalyticalX'Pert Pro a 40 kV y 40 mA con radiación Cu-K $\alpha$, entre diversos ángulos (tamaño de paso: 0,026; tiempo de conteo por paso: 8,25s). Los análisis fueron realizados por la Lic. lleana Perassi en las instalaciones de CICTERRA-CONICET.

Se realizó DRX a siete muestras, dos del piso A: T1 de cocción oxidante y antiplásticos gruesos (corte 6), y T2 de cocción reductora y antiplásticos finos (corte 7), estas muestras se corresponden con cortes delgados detallados en el acápite anterior. Dos del piso B: N1 de cocción oxidante e inclusiones finas (corte 15) y N2 de cocción reductora y antiplásticos gruesos (corte 22); y tres fragmentos experimentales de cocción oxidante y sin agregado de antiplásticos de las ubicaciones El Sunchal (M10), Camino Ancho (M12) y El Duraznillo (M19).

En todos los casos las composiciones mineralógicas fueron consistentes entre sí, siendo arcillas asignables al grupo de las illitas, y cuya temperatura de cocción sería inferior a los $800^{\circ}$ (Piñeiro 1996). Se detectó en las muestras la presencia primaria de cuarzo, plagioclasa, y feldespato (superiores en todos los casos al $97 \%$ del total), y minoritaria de moscovita, calcita y goethita generalmente en forma de trazas (menores al 1\%, figura 6).

Al respecto, la composición porcentual de las muestras experimentales demostró un elevado grado de correlación, el cual podría ser extensible a otras fuentes cercanas de arcilla, en tanto estas fueron formadas por procesos geológicos similares. Las muestras utilizadas fueron obtenidas en su totalidad de afloramientos de la formación Río Salí (caracterizada por la presencia de arcillitas, limolitas, calizas y yeso; Segemar, 2014). A su vez, las muestras arqueológicas son afines a las realizadas experimentalmente. De esta manera, los análisis de DRX indicarían la consistencia de la asociación de los fragmentos de los pisos de ocupación 
Figura 6. Gráficas comparativas de minerales identificados en DRX. A) Análisis de roca total. B) Análisis de fracción pequeña $(<2 \mu \mathrm{m})$.

Figure 6. Comparative graphics of minerals identified in DRX. A) Total rock analysis. B) Small fraction analysis $(<2 \mu \mathrm{m})$.

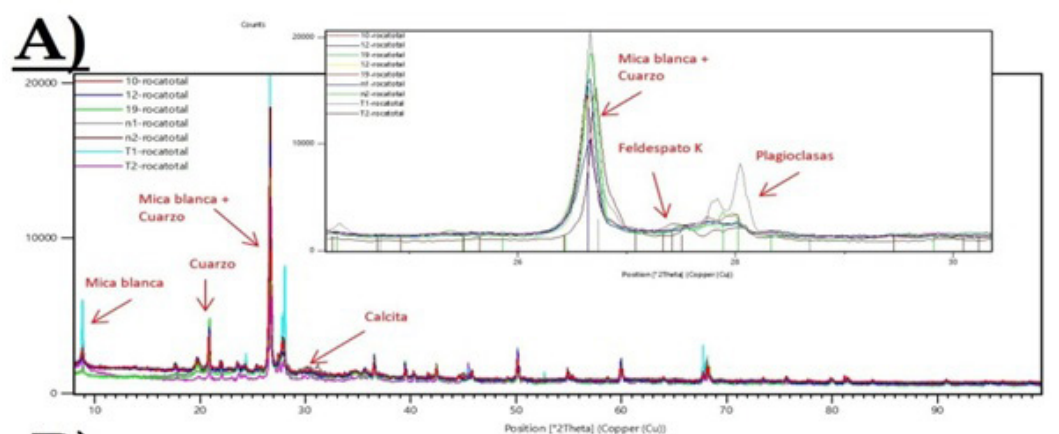

con la proveniencia local de las arcillas utilizadas en ambos.

\section{Discusión}

Los resultados aquí obtenidos resultan útiles para comparar un aspecto basal de la práctica tecno alfarera, como es el aprovisionamiento de materias primas. Al respecto, los datos de $\mathrm{El}$ Sunchal pueden confrontarse con los de sectores cercanos como El Potrerillo en el bajo de Anfama (4,5 km al sudeste de El Sunchal) y El Pedregal en el valle de La Ciénega ( $6 \mathrm{~km}$ hacia el sudoeste) donde también se han realizado análisis texturales de pastas (Cremonte 1996 y 2003; Cremonte y Pereyra Domingorena 2013).

En esos trabajos se han distinguido tres agrupamientos de fragmentos coaligados en base a análisis de clusters. El agrupamiento 1 incluye seis fragmentos recuperados en el sitio El Pedregal (valle de La Ciénega) y tres del sitio La Quebradita (valle de Tafi), y ha sido caracterizado por la presencia en todos los casos de litoclastos graníticos, abundancia de cuarzo y biotita, y en dos de los fragmentos tafinistos con presencia de litoclastos metamórficos. El agrupamiento 2 incluye ocho tiestos de La Ciénega y tres de Tafí con presencia en todos los casos de vidrio volcánico, sin inclusiones metamórficas, y eventualmente litoclastos graníticos.

Si se comparan las inclusiones de los agrupamientos 1 y 2 realizados por Cremonte con las de los fragmentos de El Sunchal se observa que ninguno sería consistente con los resultados de los pisos A y B, entre ellos, una diferencia nítida gira en torno a la presencia/ausencia de vidrio volcánico. Este tipo de inclusiones se presentarían naturalmente en algunas de las arcillas utilizadas en La Ciénega (Cremonte 2003), con lo cual deberíamos descartar la utilización de las mismas vetas por parte de los alfareros de El Sunchal.

El agrupamiento 3 incluye a un fragmento del sitio El Potrerillo y a otro denominado "Anfama gris grueso" obtenido en El Pedregal. Este tercer grupo de pastas incluye abundante proporción de litoclastos

sedimentarios y metamórficos, con presencia de cuarzo, y ausencia de biotita y vidrio volcánico. En este caso, no podrían establecerse mayores comparaciones en relación a la arcilla utilizada, pero se podría descartar la utilización de las mismas fuentes de arenas con respecto a la ocupación del piso A de El Sunchal, en tanto, la presencia de biotita y litoclastos metamórficos no ha sido allí identificada.

Sin embargo, no puede descartarse la asociación del agrupamiento 3 a la ocupación del piso B donde sí se constata la mayor presencia de litoclastos sedimentarios y metamórficos. En adición la presencia de tiestos marleados en las excavaciones de El Potrerillo indicaría una temporalidad de ocupación similar al piso B, por lo cual correspondería realizar indagaciones más exhaustivas a futuro observando la posible filiación entre las prácticas en ambas ocupaciones.

Sintéticamente, la comparación con estos sectores cercanos marcaría que en El Sunchal al igual que en otras ocupaciones del primer milenio en la región (Srur 1998 y 1999; Cremonte 1996 y 2003; Scattolin 2007; Pereyra Domingorena 2012 y 2015; Lazzari et. al. 2016 y 2017; Giusta 2017), predominaría la obtención de materias primas de origen local, a una escala posiblemente doméstica y descentralizada. Por otra parte, los valiosos aportes de Cremonte (1996 y 2003) no exploraron la posibilidad de que las variantes de los agrupamientos puedan responder a diferencias diacrónicas en relación a los modos de obtener materias primas, es decir a cambios en las tradiciones prácticas, tal como se han evidenciado en El Sunchal.

Es arriesgado aún con el grado de avance de la 
investigación disponer certezas firmes en relación a las causas y fundamentos de los cambios en la elección de materias primas. Sin embargo, una línea a explorar puede remitir a cambios en las formas de ser/habitar el espacio a lo largo del primer milenio, y manifestadas también en El Sunchal en variaciones en los patrones arquitectónicos y algunos rasgos morfoestilísticos de la cerámica ${ }^{3}$. Mientras la ocupación del piso A forma parte de un lapso de consolidación del modo aldeano/ comunitario en la región, caracterizado por la estabilidad de sus asentamientos (Berberián y Nielsen 1988; Cremonte 1996; Salazar 2011; entre otros); la del piso B forma parte de un entorno de condiciones sociales cambiantes y tal vez inestables (Scattolin 2006b; Caria y Gómez Augier 2015).

Aproximaciones realizadas desde el constructivismo estructuralista (Bourdieu 2007) a las elecciones tecnológicas de las prácticas (Gosselain 1992; Dobres y Hoffman 1994; Stark 1999; Calvo Trías y García Roselló 2014), han planteado que éstas representan fenómenos sociales en sí mismos, donde se interrelacionan aspectos materiales, sociales y simbólicos que adquieren significación en un contexto particular, y donde las elecciones tecnológicas están mediadas por interacciones sociales, sistemas de creencias, conocimientos prácticos y disponibilidad medioambiental. En adición, indagaciones etnoarqueológicas han planteado que las elecciones tecnológicas, la elección de materias primas entre ellas, son más resistentes a la variación que los aspectos decorativos (Gosselain 1992; Schiffer y Skibo 1997; Stark 1999).

En el caso de El Sunchal se podría plantear que los cambios en los tipos de arenas utilizadas pueden responder a cambios de las formas de relacionarse con el entorno y a los grados de movilidad de los asentamientos, probablemente más estables durante la ocupación del piso $A$, manifestados en inclusiones composicionalmente homogéneas; y más dinámicos durante la ocupación del piso $B$, manifestados en el uso de arenas heterogéneas. En el caso de las arcillas, esto resulta difícil de aplicar por el momento, ya que la probable fuente de obtención en ambas ocupaciones -Formación Río Salí- se extiende en dirección sur-norte a lo largo de $20 \mathrm{~km}^{2}$ aproximadamente, con afloramientos arcillosos esporádicos en los sectores bajos del valle de Anfama.

\section{Consideraciones finales}

Se han articulado análisis experimentales, petrográficos, y cristalográficos tendientes a identificar los sectores de aprovisionamiento de materias primas de dos componentes del primer milenio de la Era en El Sunchal.

\footnotetext{
${ }^{3}$ Las variaciones diacrónicas morfoestilísticas no han sido abordadas en este aporte por cuestiones de extensión. Las mismas han sido detalladas en Franco (2019a y 2019b).
}

En primer lugar, los abordajes experimentales facilitaron un primer acercamiento a las materias primas del valle de Anfama en general, y permitieron con posterioridad realizar comparaciones petrográficas y de DRX con materiales arqueológicos. En segundo lugar, los análisis petrográficos permitieron identificar materiales muy probablemente de origen local en los fragmentos de los dos pisos considerados, aunque hay una fuerte variación diacrónica de las arenas utilizadas. En tercer lugar, los estudios cristalográficos permitieron identificar el uso de arcillas consistentes con materiales locales de la formación río Salí durante ambas ocupaciones. Sintéticamente, dentro de un marco de obtención local de arcillas y arenas, se han detectado variaciones temporales en el caso de estas últimas, que permiten identificar reactualizaciones dinámicas del habitus tecnológico.

Al respecto, como plantea Sahlins (1997) la transformación cultural es una de las formas de reproducción social, en tanto las condiciones socioestructurales no son estáticas, y se actualizan en formas novedosas e impredecibles. En el caso de El Sunchal las lógicas de obtención de materias primas, al igual que en otros asentamientos coetáneos del área Sudcalchaquí no parecen haber escapado de un ámbito de decisión local/doméstico y descentralizado, sin embargo la comparación diacrónica de las mismas podría correlacionarse con cambios en las formas de habitar el espacio. Aún así, correspondería ampliar la cantidad de análisis realizados y materialidades abordadas para confirmar o afinar estas presunciones iniciales. Finalmente, este aporte apuntó a empirizar conceptos arqueológicos derivados de la teoría de la práctica, en tanto la puesta en tensión de los mismos en la experiencia práctica es un paso fundamental para evitar consideraciones apriorísticas.

Córdoba, 6 de marzo de 2020.

\section{Agradecimientos}

SECYT (UNC); SPU; CONICET; IEH; FONCyT; National Geographic Society [W464-16]; Comunidad Indígena Diaguita de Anfama; miembros y colaboradores de las campañas 20142018 del EASCC. A Olga Tarditti, Tasio Tarditti y Gilda Collo por su colaboración en distintas instancias de este trabajo. A los/as dos evaluadores/as anónimos/as que enriquecieron y mejoraron la versión original de este trabajo. Los errores y omisiones son de mi responsabilidad.

\section{Bibliografía}

Berberián, E. y Argüello de Dorsch, E. (1988). La alfarería del valle de Tafí. En Berberián, E. (Ed.), Sistemas de asentamiento prehispánicos (pp. 53-67). Córdoba, Argentina: Comechingonia.

Berberián, E. y Nielsen, A. (1988). Sistemas de 
asentamiento prehispánicos en la etapa formativa del Valle de Tafí. En Berberián, E. (Ed.), Sistemas de asentamiento prehispánicos (pp. 21-55). Córdoba, Argentina: Comechingonia.

Bourdieu, P. (2007). El sentido práctico. Buenos Aires, Argentina: Siglo XXI Editores.

Bugliani, M. F. (2010). Códigos estéticos, expresiones plásticas y modos de representación en la cerámica del Formativo en Yutopián (Valle del Cajón, Noroeste argentino). Revista del Museo de Antropología, 3, 21-32.

Calvo Trias, M. y García Roselló, J. (2014). Acción técnica, interacción social y práctica cotidiana: propuesta interpretativa de la tecnología. Trabajos de Prehistoria, 71 (1), 7-22.

Calvo Trias, M., García Rosselló, J., Javaloyas, D. y Albero, D. (2015). Playing with mud? An ethnoarchaeological approach to children's learning in Kusasi ceramic production. En Sánchez Romero, M., Alarcón, E., y Aranda Jiménez, G. (Eds.), Children, Identity and Space, (pp.88104). Oxford, Inglaterra: Oxbow.

Caria, M. y Gómez Augier, J. (2015). Arqueología en espacios contrastados en los piedemontes oriental y occidental de cumbres calchaquíes (Tucumán-Argentina) durante el $1^{\circ}$ y $2^{\circ}$ milenio de nuestra era. En Korstanje, M., Lazzari, M., Basile, M., Bugliani, M. F., Lema, V., Pereyra Domingorena, L. y Quesada, M. (Eds.), Crónicas materiales precolombinas, Arqueología de los primeros poblados del Noroeste argentino, (pp. 355-383). Buenos Aires, Argentina: Sociedad Argentina de Antropología.

Compton, R. (1962). Manual of field geology. Nueva York, Estados Unidos: John Wiley and Sons.

Cremonte, M. B. (1996). Investigaciones arqueológicas en la Quebrada de La Ciénaga (Dpto. Tafí, Tucumán) [Tesis doctoral no publicada. Facultad de Ciencias Naturales y Museo, La Plata].

Cremonte, M. B. (2003). Producción cerámica de la tradición Tafí. Estudios tecnológicos de la alfarería arqueológica de la Ciénega (Tucumán, Noroeste de Argentina). Revista do Museu de Arqueología e Etnología, 13, 57-74.

Cremonte, M. B. y Bugliani, M. F. (2009). Pasta, forma e iconografía. Estrategias para el estudio de la cerámica arqueológica. Xama, 19, 239-262.

Cremonte, M. B. y Pereyra Domingorena, L. (2013). Atlas de pastas cerámicas arqueológicas: petrografía de estilos alfareros del NOA San Salvador de Jujuy, Argentina: EdiUnJu.

Crown, P. (2001). Learning to make pottery in the Prehispanic American Southwest. Journal of Anthropological Research, 57(4), 451-469.
Di Prado, V. (2018). Prácticas alfareras prehispánicas y procesos de interacción social en el centro-este de Argentina durante el Holoceno tardío. Latin American Antiquity, 29 (3), 552-571.

Dobres, M. y Hoffman, C. (1994). Social agency and the dynamics of prehistoric technology. Journal of archaeological method and theory, 1(3), 211-258.

Feely, A. (2010). Estilos tecnológicos y tradiciones cerámicas del Bolsón de Fiambalá (Dto. Tinogasta, Catamarca) [Tesis doctoral no publicada. Facultad de Filosofía y Letras, Buenos Aires].

Franco, F. (2019a). La práctica alfarera en El Sunchal durante el primer milenio d.C. (Anfama, Tucumán). Aportes a la tradición cerámica sudcalchaquí. La Zaranda de Ideas, 17, 19-37.

Franco, F. (2019b). La práctica alfarera durante el primer milenio de la Era en El Sunchal, Anfama (Dto. Tafí Viejo, Tucumán, Rep. Argentina). Trayectorias de vida, cadenas operativas y ¿tradición? [Tesis de grado inédita. Facultad de Filosofía y Humanidades, Córdoba].

García, L. (1993). Experimentación en Inca Cueva: arcilla, fogones y combustibles. Arqueología, 3 (1), 69-91.

García Roselló, J. (2009). Cadena operativa, forma, función y materias primas. Un aporte a través de la producción cerámica del centro de Chile. Relaciones de la SAA, 34 (1), 123-148.

Giusta, M. (2017). La alfarería del primer milenio AD de la Quebrada de Amaicha (Departamento Tafí del Valle, Provincia de Tucumán): aportes sobre sus aspectos tecnológicos y potenciales materias primas. Arqueología, 23 (2), 35-61.

González, A. R. y Núñez, V. (1960). Informe preliminar sobre la investigación arqueológica en Tafí del Valle (Noroeste de Argentina). En Actas del XXXIV Congreso Internacional de Americanistas, pp. 18-25. Viena, Austria.

Gosselain, O. (1992). Technology and style: potters and pottery among Bafia of Cameroon. Man New Series, 27 (3), 559-586.

Heredia, O. (1974). Investigaciones arqueológicas en el sector meridional de las selvas occidentales. Revista del Instituto de Antropología de Córdoba, 5, 73-132.

Lazzari, M., Pereyra Domingorena, L. y Scattolin, M. C. (2016). Un paisaje social descentralizado: perspectivas integradas para comprender la producción y circulación de cerámicas en el Noroeste Argentino (Primer milenio d. C.). Actas del XIX CNAA, Serie Monográfica y Didáctica, 54, 2210-2213.

Lazzari, M., Pereyra Domingorena, L., Stoner, W., Scattolin, M. C., Korstanje, M. A. y Glascock, M. (2017). Compositional data supports decentralized model of production and circulation of artifacts in the preColumbian south-central Andes. Proceedings of the 
National Academy of Sciences, 114 (20), E3917-E3926.

Maldonado, M., Neder, L., Roldán, J. y Sampietro Vattuone, M. (2011). Caracterización geoambiental y cultural del período formativo en Selvas occidentales meridionales: sitio Horco Molle (Dpto. Yerba Buena, Tucumán). Comechingonia, 14 (1), 115-131.

Palamarczuk, V., Spano, R., Weber, F., Magnifico, D., López, S. y Manasiewicz, M. (2007). Soria 2. Apuntes sobre un sitio Formativo en el valle de Yocavil (Catamarca, Argentina). Intersecciones, 8, 121-134.

Pauketat, T. R. (2001). Practice and history in archaeology: An emerging paradigm. Anthropological Theory, 1(1), 73-98.

Pereyra Domingorena, L. (2012). Manufacturas alfareras al sur de los valles Calchaquíes entre el siglo primero al quinto DC. Relaciones de la SAA, 37 (2), 387-412.

Pereyra Domingorena, L. (2013). Cerámica experimental con materias primas del sur de los valles Calchaquíes (Noroeste argentino). Intersecciones, 14, 239-250.

Pereyra Domingorena, L. (2015). Estudio petrográfico de la cerámica arqueológica del primer milenio d.C. al sur de los Valles Calchaquíes (Noroeste Argentino). Chungara, 47 (3), 415-428.

Pérez Pieroni, J. (2017). Pastas cerámicas y muestras de arenas: comparación petrográfica y aproximación a la obtención de materias primas en la cuenca sur de Pozuelos (Jujuy, Argentina). Arqueología, 23 (1), 11-25.

Piñeiro, M. (1996). Manejo de recursos y organización de la producción cerámica en Rincón Chico, Catamarca. Relaciones de la SAA, 21 (1), 161-185.

Sahlins, M. (1997). Islas de Historia, La muerte del capitán Cook, Metáfora, Antropología e Historia. Barcelona, España: Gedisa.

Salazar, J. (2011). Reproducción social doméstica y asentamientos residenciales entre el 200 y el 800 d.C. en el valle de Tafí, Provincia de Tucumán. [Tesis doctoral no publicada. Facultad de Filosofía y Humanidades, Córdoba].

Salazar, J., Franco Salvi, V., Berberián, E. y Cravero, S. (2008). Contextos domésticos del valle de Tafí, Tucumán, Argentina (200-1000 AD). Werken, 10, 25-47.

Salazar, J., Molar, R, Montegú, J., Franco, F., Vázquez Fiorani, A., Moyano, G., Chiavassa Arias, S., Carrasco, D. y Franco Salvi, V. (2019). Investigaciones arqueológicas en la cuenca de Anfama, provincia de Tucumán. En Laguens,
A., Bonnin, M. y Marconetto, B. (Compiladores), Actas del XX Congreso Nacional de Arqueología Argentina, (pp. 195-199). Córdoba, Argentina: UNC.

Scattolin, M. C. (2006a). Contornos y confines del universo iconográfico precalchaquí del valle de Santa María. Estudios Atacameños, 32 (1), 119-139.

Scattolin, M. C. (2006b). De las comunidades aldeanas a los curacazgos. Boletín de Arqueología PUCP, 10, 357-398.

Scattolin, M. C. (2007). Santa María antes del año mil. Fechas y materiales para una historia cultural. En Williams, V., Ventura, B., Callegari, M. y Yacobaccio, H. (Eds.), Sociedades precolombinas surandinas. Temporalidad, interacción y dinámica Cultural del NOA en el Ámbito de los Andes CentroSur, (pp. 203-220). Buenos Aires, Argentina: Buschi.

Scattolin, M. C., Pereyra Domingorena, L., Cortés, L., Bugliani, M. F., Calo, M., Izeta, A. y Lazzari, M. (2007). Cardonal: una aldea formativa entre los valles y la puna. Cuadernos de la UNJu, 32 (1), 211-225.

Schiffer, M. y Skibo, J. (1997). The explanation of artifact variability. American Antiquity, 62 (1), 27-50.

Schuster, V. (2014). La organización tecnológica de la cerámica de cazadores-recolectores. Costa Norte de la provincia del Chubut (Patagonia, Argentina). Relaciones de la SAA, 39 (1), 203-231.

Segemar (2014). Hoja Geológica 2766-Il Tucumán. Buenos Aires: Instituto de Geología y Recursos Minerales.

Srur, F. (1998). Análisis de la cerámica arqueológica del montículo, sitio Casas Viejas, Dpto. Tafí del Valle, Tucumán [Tesis de grado no publicada. Facultad de Ciencias Naturales e Instituto Miguel Lillo, Tucumán].

Srur, F. (1999). Cerámica de la fase 1 de la tradición Tafí. Actas del XIII Congreso Nacional de Arqueología Argentina, Tomo 1, 180a-180g. Córdoba.

Stark, M. (1999). Social dimensions of technical choice in Kalinga ceramic traditions. En Chilton, E. (Ed.), Material meanings: Critical approaches to the interpretations of material culture, (pp. 24-43). Salt Lake City, Estados Unidos, University of Utah Press.

Vidal, A. (2019). Nadie nace sabiendo: los aprendices de la cerámica hecha a mano. Treballs d'Arqueologia, 23, 237-257.

Wenthworth, C. (1922). A scale of grade and class terms for clastic sediments. The Journal of Geology, 30 (5), 377-392. 